\title{
NMMR Studies of an Immunomodulatory Benzodliazepine Binding to its Molecular Target on the Mitochondrial $F_{1} F_{0}-A T P a s e$
}

Andrew C. Stelzer, ${ }^{1}$ Richard W. Frazee, ${ }^{2}$ Chad Van Huis, ${ }^{3}$ Joanne Cleary, ${ }^{2}$

Anthony W. Opipari Jr., ${ }^{4}$ Gary D. Glick, ${ }^{2}$ Hashim M. Al-Hashimi ${ }^{1}$

${ }^{1}$ Department of Biophysics, University of Michigan, Ann Arbor, MI 48109

${ }^{2}$ Department of Chemistry, University of Michigan, Ann Arbor, MI 48109

${ }^{3}$ Lycera Corporation, 46701 North Commerce Center Drive, Plymouth, MI 48170

${ }^{4}$ Department of Obstetrics and Gynecology, University of Michigan, Ann Arbor, MI 48109

Received 13 August 2009; revised 24 August 2009; accepted 24 August 2009

Published online 18 September 2009 in Wiley InterScience (www.interscience.wiley.com). DOI 10.1002/bip.21306

\section{ABSTRACT:}

$B z-423$ is an inhibitor of the mitochondrial $F_{1} F_{0}$-ATPase, with therapeutic properties in murine models of immune diseases. Here, we study the binding of a water-soluble Bz-423 analog (5-(3-(aminomethyl)phenyl)-7-chloro1-methyl-3-(naphthalen-2-ylmethyl)-1Hbenzo][e][1,4]diazepin-2(3H)-one); (1) to its target subunit on the enzyme, the oligomycin sensitivity conferring protein (OSCP), by NMR spectroscopy using chemical shift perturbation and cross-relaxation experiments. Titration experiments with constructs representing residues $1-120$ or 1-145 of the OSCP reveals that (a) 1 binds to a region of the protein, at the minimum, comprising residues M51, L56, K65, V66, K75, K77, and N92, and (b) binding of 1 induces conformational changes in the OSCP. Control experiments employing a variant of 1 in which a key binding element on the small molecule was deleted; it had no perturbational effect on the spectra of the OSCP, which indicates that the observed changes with 1

Additional Supporting Information may be found in the online version of this article.

Correspondence to: Gary D. Glick; e-mail: gglick@umich.edu or Hashim M. AlHashimi; e-mail: hashimi@umich.edu Contract grant sponsor: NIH

Contract grant number: AI-047450

(c) 2009 Wiley Periodicals, Inc. represent specific binding interactions. Collectively, these data suggest that 1 might inhibit the enzyme through an allosteric mechanism where binding results in conformational changes that perturb the OSCP- $F_{1}$ interface resulting in disrupted communication between the peripheral stalk and the $F_{1}$-domain of the enzyme. (C) 2009 Wiley Periodicals, Inc. Biopolymers 29: 85-92, 2010.

Keywords: binding; allosteric; bioener

This article was originally published online as an accepted preprint. The "Published Online" date corresponds to the preprint version. You can request a copy of the preprint by emailing the Biopolymers editorial office at biopolymers@wiley. com

\section{INTRODUCTION}

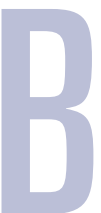

z-423 is a 1,4-benzodiazepine that potently suppresses disease in autoimmune mice by selectively killing pathogenic lymphocytes. ${ }^{1,2}$ Affinity-based screening of a phage-display human cDNA expression library identified the oligomycin-sensitivity conferring protein (OSCP), a component of the mitochondrial $\mathrm{F}_{1} \mathrm{~F}_{0}$-ATPase, as the molecular target for $\mathrm{Bz}-423 .{ }^{3}$ Binding of Bz-423 to the OSCP in the context of intact enzyme inhibits both synthesis and hydrolysis of ATP. ${ }^{3,4}$ Consistent with modulation of $\mathrm{F}_{1} \mathrm{~F}_{0}$-ATPase activity, Bz-423 increases the generation of superoxide from the mitochondrial respiratory chain and this reactive oxygen species, rather than 

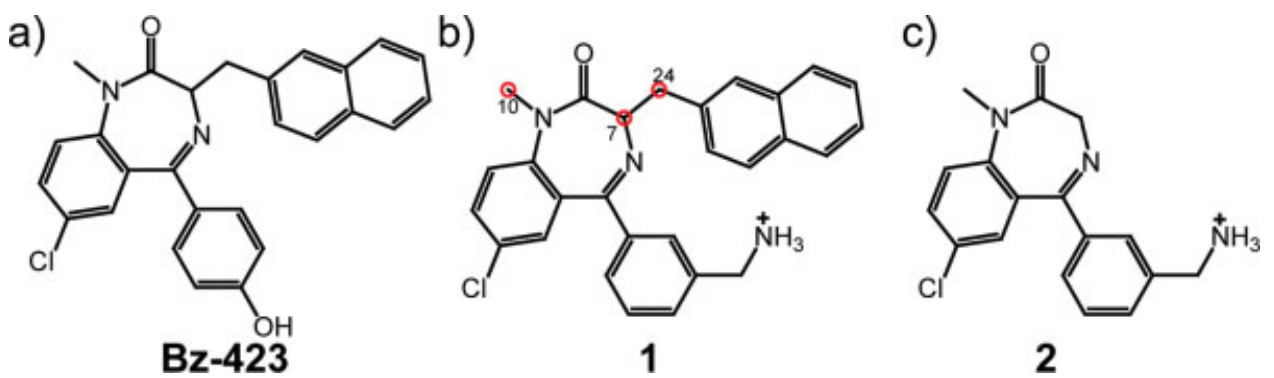

FIGURE 1 Chemical structure of (a) Bz-423 and the molecules used in this study: (b) 5-(3-(aminomethyl)phenyl)-7-chloro-1-methyl-3-(naphthalen-2-ylmethyl)-1H-benzo[e] [1,4]diazepin-2(3H)one (1) and (c) (aminomethyl)phenyl)-7-chloro-1-methyl-1H-benzo[e][1,4]diazepin-2(3H)-one (2). Protons of 1 that were saturated in cross-relaxation experiments are labeled. Based on prior structure-reactivity studies as a guide, 1 was designed to replace the critical phenolic proton with an ammoninum group to enhance aqueous solubility. The activity of 1 in enzyme and cellular assays is comparable. Removing the naphthyl group in $\mathbf{2}$ abolishes all activity against the enzyme.

changes in ATP concentration, is the signal that initiates apoptosis. $^{5,6}$

The OSCP is a 213 amino acid long protein (including the mitochondrial leader sequence) that is conserved among mammals and is not present in other ATPases. ${ }^{7}$ The OSCP along with subunits $b$, $d$, and $F_{6}$ form the peripheral stalk in mammalian $\mathrm{F}_{1} \mathrm{~F}_{0}$-ATPases. ${ }^{8}$ The stalk links the integral membrane $\mathrm{F}_{0}$ component of the enzyme with its soluble catalytic $\mathrm{F}_{1}$ domain, which is located in the mitochondrial matrix. The peripheral stalk is believed to act as a stator, holding the $\mathrm{F}_{1} \quad \alpha_{3} \beta_{3}$ hexamer static while the central stalk $\left(\gamma \delta \varepsilon \mathrm{c}_{10}\right)$ rotates. ${ }^{9,10}$ To function properly, the stalk subunits must act in concert with one another; while the peripheral stalk subunits do not need to move in relation to one another during catalysis, disrupting the connections between the subunits disrupts coupling between $\mathrm{F}_{0}$ and $\mathrm{F}_{1} \cdot{ }^{11}$ In addition, the stalk must be anchored at each end for proper function: the transmembrane domain of subunit b holds the stalk in the mitochondrial membrane, while the N-terminal end of the OSCP sits on top of the $\alpha_{3} \beta_{3}$ hexamer with the C-terminus protruding almost $100 \AA$ along the surface of $\mathrm{F}_{1}$ towards $\mathrm{F}_{0} \cdot{ }^{12}$ The $\mathrm{N}$-terminal tails of the $\alpha$-subunits are critical for binding to OSCP. ${ }^{13}$

The structure of a 134-amino acid long N-terminal fragment of the $\delta$-subunit from E. coli, the bacterial homolog of the bovine OSCP, has been studied by NMR spectroscopy. ${ }^{14}$ The protein adopts a 6-helix bundle with a disordered C-terminus. NMR studies using the N-terminal domain of the bovine OSCP (OSCP-NT, residues 1-120) reveals a similar fold. ${ }^{15,16}$ Binding experiments with peptide fragments from the $\mathrm{N}$-termini of $\mathrm{F}_{1} \alpha$-subunits suggests that the interaction site on OSCP-NT comprises a hydrophobic groove between helices I and V. Hence, this interface, which is essential for the rotary mechanism of the enzyme, probably consists of helix-helix interactions.

Because Bz-423 does not bind at the active site of enzyme, we hypothesized that it may bind at, or near the $\mathrm{F}_{1}$-OSCP interface where it can perturb one or more of the conformational transitions associated with the rotary (bindingchange) mechanism of catalysis. ${ }^{4}$ To gain support for this hypothesis, we studied the binding of water soluble Bz-423 analogs (Figure 1) with OSCP constructs representing amino acids $1-120$ and 1-145 by NMR spectroscopy using chemical shift perturbation and cross-relaxation experiments to localize the binding site. Our data identified a recognition site near the $\mathrm{F}_{1}$-OSCP interface and a conformational change in the protein upon drug binding. Collectively, these data suggest that Bz-423 like inhibitors may function in an allosteric manner inducing a conformational change that hinders catalysis.

\section{MATERIALS AND METHODS}

\section{Small Molecules Ligands}

Benzodiazepines $\mathbf{1}$ and $\mathbf{2}$ were synthesized and characterized as previously described. ${ }^{15}$ Samples of both compounds used in the NMR titration experiments were $>95 \%$ pure.

\section{Plasmid Construction}

A 360-base-pair long insert encoding a truncated bovine OSCP, containing amino acids $1-120$, was prepared via PCR using forward $\left(5^{\prime}-\right.$ CACCATGTTTGCCAAGCTTGTGAGGCC- $3^{\prime}$ ) and reverse (5'-CTA AACTGTGCATGGTACTTCTCC- $\left.3^{\prime}\right)$ oligonucleotide primers $(25 \mu \mathrm{M}$ each) in the presence of dNTPs $(0.5 \mathrm{mM})$, pOSCP (1 ng), PFU Turbo DNA polymerase (3-5 U; Stratagene, La Jolla, CA), and $1 \times \mathrm{PFU}$ Turbo DNA polymerase buffer. PCR conditions consisted of $30 \mathrm{~s}$ at 
$94^{\circ} \mathrm{C}, 30 \mathrm{~s}$ annealing at $55^{\circ} \mathrm{C}$, and a $1 \mathrm{~min}$ extension at $72^{\circ} \mathrm{C}$ for 31 cycles. ${ }^{17}$ A 435-base-pair long insert encoding a truncated bovine OSCP containing amino acids $1-145$ was prepared in a similar manner using the same forward oligonucleotide primer as above together with a reverse ( $5^{\prime}$-CTGGCCTTTACTTAGGAAGCTCTTCAGG- $3^{\prime}$ ) oligonucleotide primer. The PCR products were purified using a Qiagen (Valencia, CA) PCR Clean Up kit following the manufacturer's instructions. Both inserts were cloned into expression vectors provided in the TOPO 10 kit following the manufacture's instructions (Invitrogen, Carlsbad, CA). The OSCP 1-120 insert was ligated into pCRT7/NT-TOPO that contains both an Express ${ }^{\mathrm{TM}}$ Epitope coding sequence and a hexa-his tag, placing the OSCP 1-120 coding region C-terminal to these sequences. The OSCP 1-145 insert was ligated into pCRT7/CT-TOPO, which contains both a V5 Epitope coding sequence and a hexa-his tag, placing the OSCP 1-145 coding region $\mathrm{N}$-terminal to these sequences. Thus, OSCP120 contains an N-terminal hexa-his tag connected by the linker sequence GMASMTGGQQMGRDLYDDDDKDPTL, while the OSCP145 construct contains a C-terminal hexa-his tag connected by the linker sequence KGNSKLEGKPIPNPLLGLDSTRTG. Primary structures for all OSCP constructs are shown in Supporting Information Figure S1. Ligation products were transformed into TOP-10 cells and selected on LBampicillin $(50 \mu \mathrm{g} / \mathrm{mL})$ plates. Colonies were screened for inserts via PCR using mini-prep plasmid DNA and both the forward and reverse T7 primers. The presence of the correct inserts was confirmed by automated DNA sequencing (Sequencing Core Facility, University of Michigan, Ann Arbor, MI).

\section{Growth and Expression of OSCP Constructs}

OSCP constructs pCRT7/NT-OSCP120 and pCRT7/CT-OSCP145 were transformed into One Shot BL21(DE3) pLysS (Invitrogen, Carlsbad, CA) using the manufacturer's procedure. Individual colonies were picked immediately to inoculate $1-10 \mathrm{~mL}$ LB overnight cultures supplemented with ampicillin $(200 \mu \mathrm{g} / \mathrm{mL})$. Cultures were incubated at $37^{\circ} \mathrm{C}$ with rotary shaking at $250 \mathrm{rpm}$. One-liter cultures containing either Spectra 9-N $\left(>98 \%{ }^{15} \mathrm{~N}\right)$, Spectra $9-\mathrm{CN}\left(>98 \%{ }^{15} \mathrm{~N},>98 \%\right.$ ${ }^{13} \mathrm{C}$, or Spectra $9-\mathrm{dCN}\left(>97 \% \mathrm{D}_{2} \mathrm{O},>98 \%{ }^{15} \mathrm{~N},>98 \%{ }^{13} \mathrm{C}\right)$ media (Spectra Stable Isotopes, Columbia, molecular dynamics (MD)), were supplemented with ampicillin $(200 \mu \mathrm{g} / \mathrm{mL})$ and inoculated with the overnight culture. Cultures were grown at $37^{\circ} \mathrm{C}$ with rotary shaking at $250 \mathrm{rpm}$ until $\mathrm{A}_{595}$ reached 0.6-1 (Spectra 9-dCN required double the growth time compared to the other media) at which point cultures were removed to $4^{\circ} \mathrm{C}$ while the rotary shaker temperature was brought down to $20^{\circ} \mathrm{C}$ using a heat exchanger over a period of $1 \mathrm{~h}$. IPTG was added to a final concentration of $0.02 \mathrm{mM}$. Growth and expression continued for $20 \mathrm{~h}$ at $20^{\circ} \mathrm{C}$. Cell pastes were harvested via centrifugation in a Beckman JLA8.1 at $6000 \mathrm{~g}$ for $10 \mathrm{~min}$ at $4^{\circ} \mathrm{C}$. Cell pastes were resuspended in ice-cold Nickel NTA buffer $(25 \mathrm{~mL} ; 50 \mathrm{mM}$ Tris- $\mathrm{HCl}$ $\mathrm{pH} 8,300 \mathrm{mM} \mathrm{NaCl}, 0.001 \%$ phenylmethanesulfonyl fluoride, and Roche Complete protease inhibitors w/o EDTA as per manufacturers instruction). The suspension was transferred to a $50 \mathrm{~mL}$ Falcon tube on ice and stored at $-80^{\circ} \mathrm{C}$.

\section{Purification of OSCP 120NT (His Tag) and OSCP 145CT (His Tag)}

Cell paste was thawed at room temperature and placed immediately on ice. The suspension was transferred to a $50 \mathrm{~mL}$ beaker on ice and sonicated using the standard horn (set at output $=8.5$ ) for 620 -s long intervals between which was a 2-min long rest on ice to dissipate heat. After sonication, the mixture was transferred to two Beckman JA25.5 centrifuge tubes and centrifuged at $21,000 \mathrm{rpm}$ at $4{ }^{\circ} \mathrm{C}$ for $45 \mathrm{~min}$. The soluble extract was loaded $(0.5 \mathrm{~mL} / \mathrm{min})$ onto a Ni-NTA affinity column $(1 \mathrm{~cm}$ diameter $\times 5 \mathrm{~cm}$ long) equilibrated in Nickel NTA Buffer at $4{ }^{\circ} \mathrm{C}$. The column was washed with Nickel NTA Buffer$25 \mathrm{mM}$ imidazole $(25 \mathrm{~mL})$, and protein was eluted with Nickel NTA Buffer- $250 \mathrm{mM}$ imidazole $(25 \mathrm{~mL})$. Fractions of about $1 \mathrm{~mL}$ were collected and those from fractions 3-14 were direct placed into snakeskin dialysis tubing (7000 MWCO, Pierce, Rockville, IL) immersed in of OSCP Buffer (4 L; 50 mM Tris-Cl pH 8, 30 mM NaCl, 1 mM EDTA, $5 \mathrm{mM} \beta$-mercaptoethanol). Complete protease inhibitors (with or without EDTA) were added following the manufacturer's instructions, directly to the fractions in the dialysis bag. Following overnight dialysis at $4{ }^{\circ} \mathrm{C}$ with stirring, the dialysate was cleared by centrifugation using a Beckman JA 25.5 rotor for $20 \mathrm{~min}$ at $21,000 \mathrm{rpm}$ and $4^{\circ} \mathrm{C}$. The clarified extract was loaded into a ÄKTA prime super loop $(50 \mathrm{~mL})$ at $4{ }^{\circ} \mathrm{C}$ and injected at $1.3 \mathrm{~mL} / \mathrm{min}$ onto a $5 \mathrm{~mL}$ HiTrap SpHP column (Pharmacia) equilibrated in at least five volumes of modified OSCP Buffer (50 mM Tris-Cl pH 8, $30 \mathrm{mM} \mathrm{NaCl}, 1 \mathrm{mM}$ EDTA, 0.001\% phenylmethanesulfonyl fluoride, Roche Complete protease inhibitors w/o EDTA, $5 \mathrm{mM} \beta$-mercaptoethanol; MOB) at $4^{\circ} \mathrm{C}$. The column was washed with two bed volumes of the same buffer and then eluted with a $75 \mathrm{~mL}$ linear gradient $(\mathrm{MOB}$ and $\mathrm{MOB}+500 \mathrm{mM} \mathrm{NaCl})$ at $1 \mathrm{~mL} /$ min while collecting $1 \mathrm{~mL}$ fractions. OSCP $120 \mathrm{NT}$ eluted in a sharp band centered around $250 \mathrm{mM} \mathrm{NaCl}$, while OSCP 145CT eluted in a similar manner at $280 \mathrm{mM} \mathrm{NaCl}$.

\section{NMR Spectroscopy}

All NMR experiments were performed at $25^{\circ} \mathrm{C}$ unless indicated otherwise using an Avance Bruker $600 \mathrm{MHz}$ spectrometer equipped with a $5 \mathrm{~mm}$ triple-resonance cryogenic probe. NMR spectra were processed and analyzed using NMRPipe and SPARKY 3. ${ }^{18,19}$ The NMR buffer consisted of $90 / 10 \% \mathrm{H}_{2} \mathrm{O} / \mathrm{D}_{2} \mathrm{O}$ containing $50 \mathrm{mM}$ Tris, $5 \mathrm{mM} \mathrm{KCl}$, $5 \mathrm{mM} \beta$-mercaptoethanol, $0.001 \%$ PMSF, and protease inhibitor cocktail at $\mathrm{pH} \sim 7$. For cross-relaxation experiments, a ${ }^{2} \mathrm{H} /{ }^{15} \mathrm{~N}$ labeled OSCP120 $(0.3 \mathrm{mM})$ sample was used in an NMR buffer consisting of $90 / 10 \% \mathrm{D}_{2} \mathrm{O} / \mathrm{H}_{2} \mathrm{O}$ containing deuterated TRIS $(50 \mathrm{mM})$ and deuterated $\beta$-mercaptoethanol $(5 \mathrm{mM})$ at $\mathrm{pH} \sim 7$ without PMSF and protease inhibitor cocktail to minimize spectral overlap with $1.93 \%$ of the OSCP120 backbone amides could be assigned based on a previous NMR study (BMRB entry 6564) (Supplementary Information Figure S2) ${ }^{20}$ The OSCP120 resonances in OSCP145 were assigned by overlaying spectra and using standard triple resonance experiments on a doubly labeled $\left({ }^{13} \mathrm{C} /{ }^{15} \mathrm{~N}\right)$ OSCP145 sample $(0.5 \mathrm{mM})$.

Two-dimensional (2D) ${ }^{1} \mathrm{H}_{-}{ }^{15} \mathrm{~N}$ HSQC spectra of ${ }^{15} \mathrm{~N}$ labeled OSCP were recorded following incremental addition of $\mathbf{1}$ from a stock solution $(20 \mathrm{mM})$ in NMR buffer for protein:ligand ratios of $1: 1,1: 2,1: 4$, and $1: 8$. Weighted average chemical shift perturbations were calculated using,

$$
\Delta_{\mathrm{av}} N H=\sqrt{\frac{(\delta H)^{2}+(\delta N / 5)^{2}}{2}}
$$

where $\delta H$ and $\delta N$ are the proton and nitrogen amide chemical shift values in ppm. Weighted average chemical shifts $>0.01 \mathrm{ppm}$ were considered significant. 

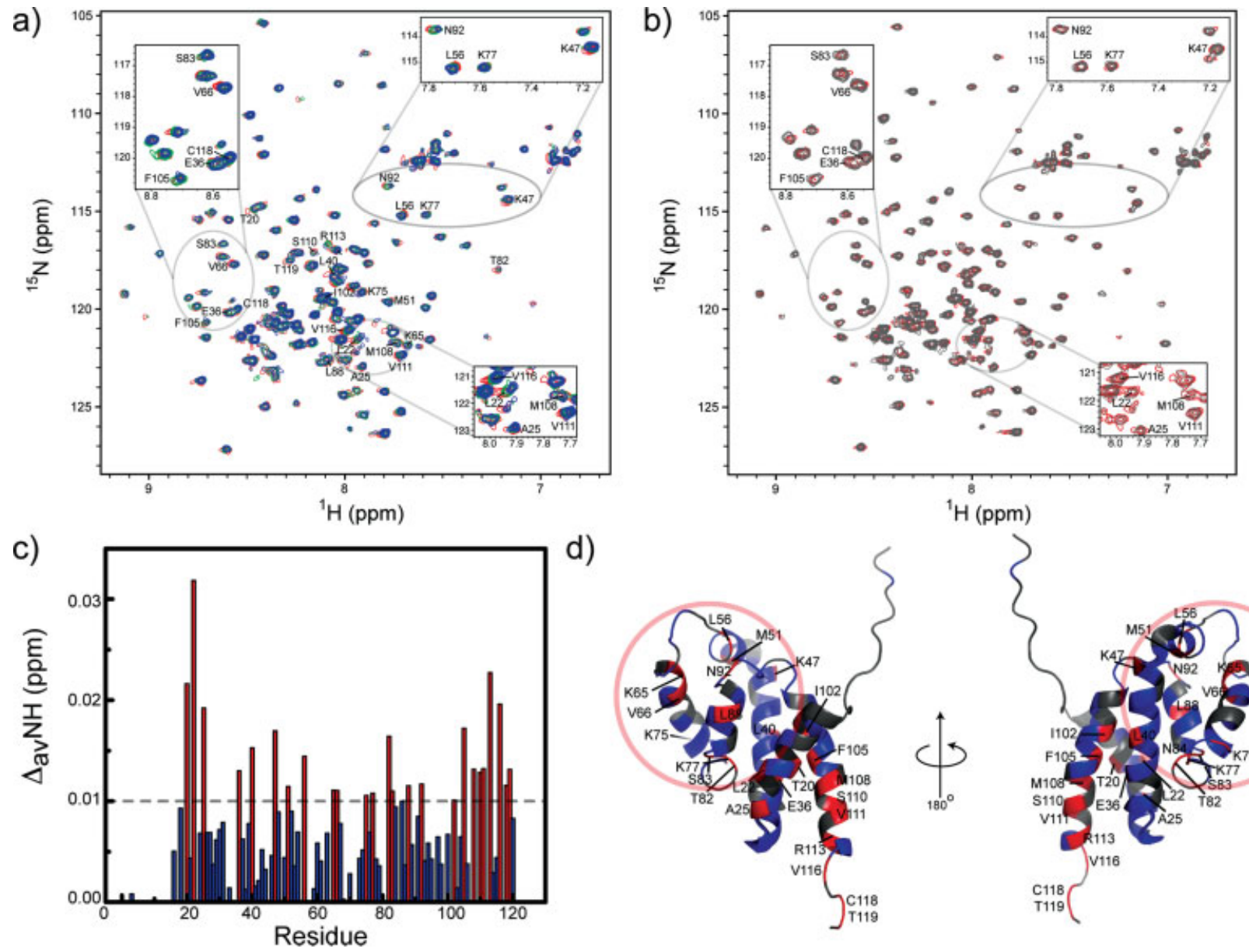

d)
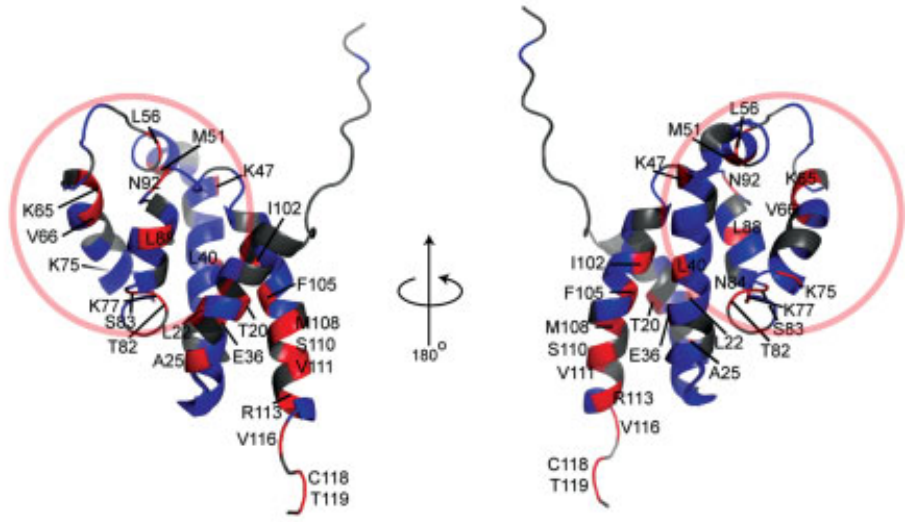

FIGURE 2 Chemical shift perturbations from titrations of 1 and 2 onto OSCP120 are shown in (a) and (b), respectively. Free OSCP120 (red), 1:2 OSCP120:1, ${ }^{35}$ 1:4 OSCP120:1 (blue), and 1:4 OSCP120:2 (gray) spectra are shown. Only resonances that exhibited significant chemical shift perturbations are labeled in (a), and peaks labeled in (b) are for comparison purposes. Weighted average of chemical shift perturbations (c) are color coded to match coloring on the OSCP120 structure (d) with blue representing resonances that showed no significant perturbation and red showing those resonances with shifts greater than the threshold of $0.01 \mathrm{ppm}$. Residues that could not be monitored are colored in gray and the shoulder region is circled in (d). Protein precipitation was observed at 1:8 OSCP:ligand ratios, which precluded further increasing the ligand concentration.

Cross-relaxation experiments were performed at $14^{\circ} \mathrm{C}$ to improve cross-relaxation efficiency by modifying a $2 \mathrm{D}{ }^{1} \mathrm{H}-{ }^{15} \mathrm{~N}$ HSQC experiment from the Bruker pulse program library (hsqcfpf3gpphwg). ${ }^{21}$ A $1.8 \mathrm{~s}$ adiabatic Wurst pulse was applied following a predelay of $1.4 \mathrm{~s}$ for saturating one of three different ligand proton resonances (protons 7, 10, and 24; Figure 1) with a total saturation bandwidth of $\sim 30 \mathrm{~Hz}^{22}$ An additional reference experiment was also recorded with off-resonance $(-50 \mathrm{ppm})$ saturation. Each experiment required $\sim 23 \mathrm{~h}$ of acquisition time. Peak intensity errors were calculated using NMRPipe and ranged between $4 \%$ and $19 \% .{ }^{18,19}$ Cross-relaxation intensity ratios were calculated by taking the ratio of peak intensities measured with on-resonance $\left(I_{\text {sat }}\right)$ and off-resonance $\left(I_{\mathrm{o}}\right)$ saturation. Only well-resolved resonances with a signal-to-noise ratio $>10$ were analyzed. Chemical shift perturbations and cross-relaxation results were mapped onto OSCP structures and visualized using Pymol. ${ }^{23}$

\section{RESULTS}

\section{Mapping the Binding of 1 to OSCP120 Using Chemical Shift Perturbation}

In a first group of studies, we attempted chemical shift perturbation experiments using full-length, ${ }^{15} \mathrm{~N}$ labeled OSCP (OSCP190) (Supporting Information Figure S1). However, the 2D HSQC spectra of OSCP190 were intractable due to aggregation identified by uniform reduction in NMR signal intensities. ${ }^{16,20}$ We subsequently prepared two truncated constructs for binding measurements. The first protein comprised residues 1-120 (OSCP120) and has been studied previously by $\mathrm{NMR}$, and the second construct contained residues $1-145$ (OSCP145). Titration of 1 into ${ }^{15} \mathrm{~N}$ labeled 
OSCP120 at protein:ligand ratios of 1:1, 1:2, 1:4, and 1:8 led to chemical shift perturbations that suggest rapid exchange on the NMR timescale consistent with micromolar affinity, which agreed with previous data for Bz-423 (Figures 1 and 2 and Supplementary Information Table S1). ${ }^{4}$ To assess the significance of the OSCP chemical shift perturbations, a second set of titration experiments were conducted using an analog lacking the naphthalene substituent 2 . This analog possesses no activity in the $\mathrm{F}_{1} \mathrm{~F}_{0}$-ATPase or cell-based assays, (data not shown) and therefore, we hypothesized should not to bind to the protein. Indeed, chemical shift perturbations were not observed in titration experiments with 2 (Figure 2), indicating that the perturbations observed with 1 reflect specific binding interaction(s) that presumably are related to its inhibitory activity.

The chemical shift perturbations induced by 1 were distributed at different sites within the OSCP120 construct (Figure 2). This observation suggests that 1 either binds at multiple sites and/or binding at one site causes conformational changes elsewhere in the protein. The range of intensities observed in OSCP120 suggests that the protein is highly flexible and potentially prone to ligand-induced (allosteric) conformational changes. In addition, previous studies of $\mathrm{Bz}$ 423 suggest a 1:1 stoichiometry upon binding to the $\mathrm{F}_{1} \mathrm{~F}_{0^{-}}$ATPase. ${ }^{4}$ Collectively, these observations are consistent with ligand-induced conformational changes away from the binging site.

The chemical shift perturbations caused by $\mathbf{1}$ fall in three general regions of OSCP120; one is located between helices III, IV, and V ("shoulder") and includes residues M51, L56, K65, V66, K75, K77, T82, S83, and N92; the second is located at the C-terminal tails of helices I and VI ("tail region") and includes residues V111, R113, V116, C118, and T119; a third potential locus is located between the "tail" and "shoulder" regions (Figure 3). Interestingly, the latter region includes residues A25 and L88, which were previously shown to interact directly with the $\alpha$ peptide mimicking the $\mathrm{F}_{1}$ domain of the $\mathrm{F}_{1} \mathrm{~F}_{0}$-ATPase. ${ }^{16,20}$

To confirm the data obtained with OSCP120, we used a second OSCP construct comprising 145 amino acids (OSCP145). Unlike the OSCP190, this truncated protein did not aggregate and was amenable to chemical shift perturbation experiments with 1. Comparing the OSCP120 and OSCP145 spectra reveals that the majority of the OSCP120 resonances are not altered due to the additional 25 amino acids in the OSCP145 construct (Supplementary Information Figure S3). Not surprisingly, residues that showed significant differences between the two constructs were primarily located at the $\mathrm{N}$ - and C-termini. However, chemical shift differences were observed at helix $\mathrm{V}$, which interacts with the $\mathrm{F}_{1}$ peptide. ${ }^{16}$ This region, which also undergoes perturbations

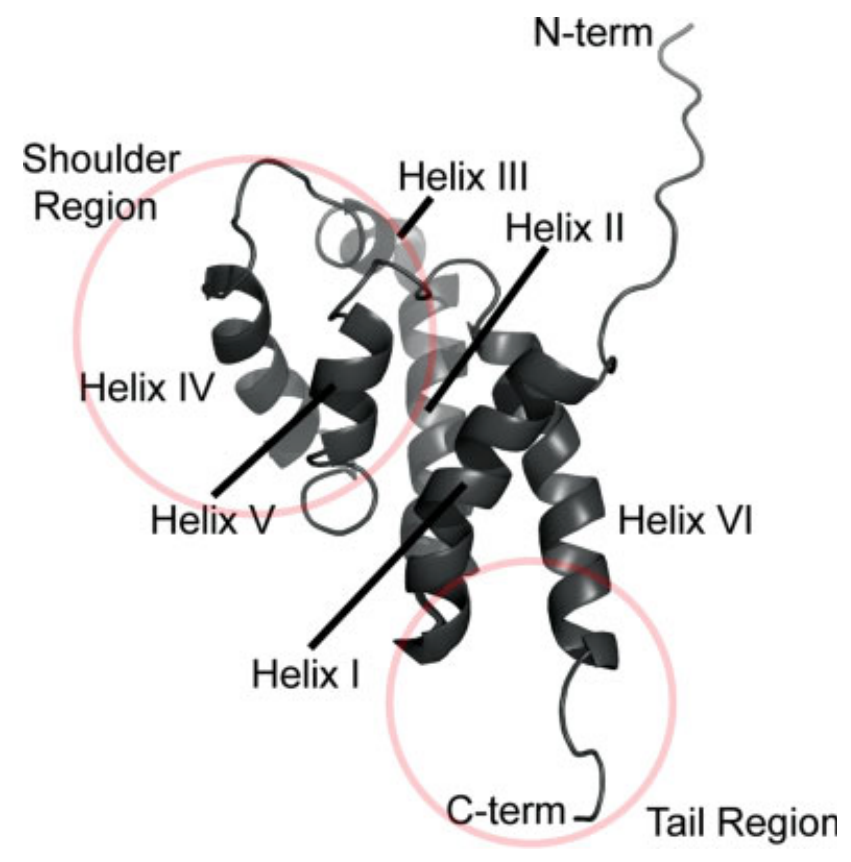

FIGURE 3 The structure of OSCP120 (PDB ID\# 2BO5) is shown with helices, termini, shoulder, and tail regions labeled.

with 1, may be subject to allosteric conformational perturbations that could be important in mediating OSCP interactions with the $\mathrm{F}_{1} \mathrm{~F}_{0}$-ATPase $\mathrm{F}_{1}$ domain. ${ }^{4}$

Titration with 1 induced chemical shift perturbations in OSCP145, whereas 2 did not (Supplementary Information Figure S4). Several resonances from the shoulder (M51, L56, K65, V66, $\mathrm{K} 75$, and N92) and middle region of the protein (A25, E36, L40, K47, I102, and F105) that overlaid in OSCP120 and OSCP145 showed similar perturbations with 1, including A25, which interacts with the $\mathrm{F}_{1}$ helix (Supplementary Information Figure S3). Many residues in the shoulder region (E48, A52, K67, T73, S79, S83, E91, and R94) also showed larger perturbations with 1 in OSCP145 compared to OSCP120. By contrast, some of the perturbations observed in the "tail" (residues V116 and C118), middle (L88), and shoulder (T82) regions were less pronounced in OSCP145. Taken together, these data indicate that the shoulder region is the most probable binding site for $\mathbf{1}$ on OSCP.

\section{Probing the OSCP Ligand-Binding Site}

\section{by Cross-relaxation}

Because chemical shift perturbations can arise from binding and/or conformational changes, cross-relaxation experiments were employed to localize the binding site of $\mathbf{1}$ on OSCP $120 .^{24}$ For these studies, 2D HSQC spectra of OSCP following saturation of specific ${ }^{1} \mathrm{H}$ resonances in $\mathbf{1}$, and an equivalent reference spectrum with off-resonance $(-50 \mathrm{ppm})$ saturation were measured. A reduction in OSCP resonance 
a)
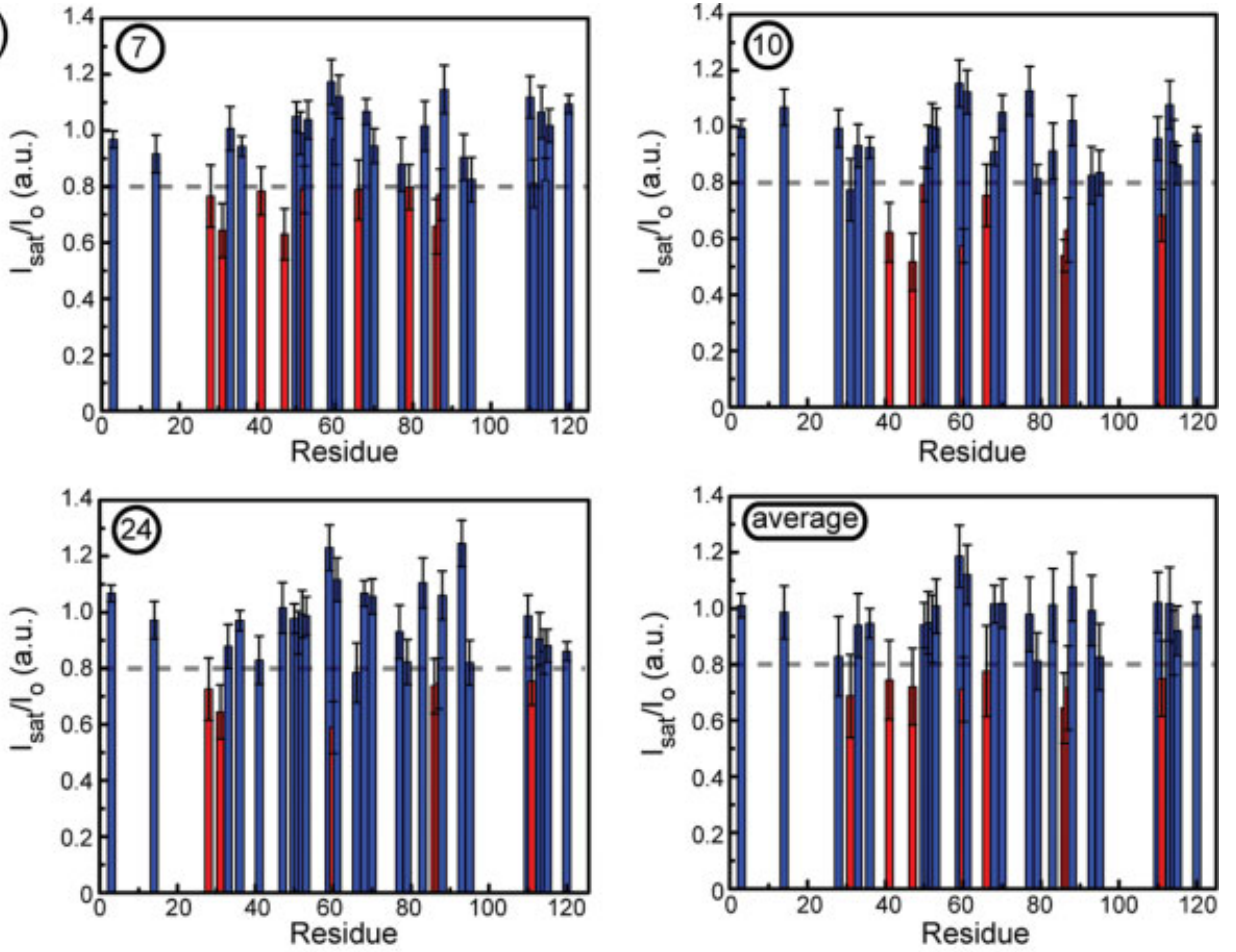

b)

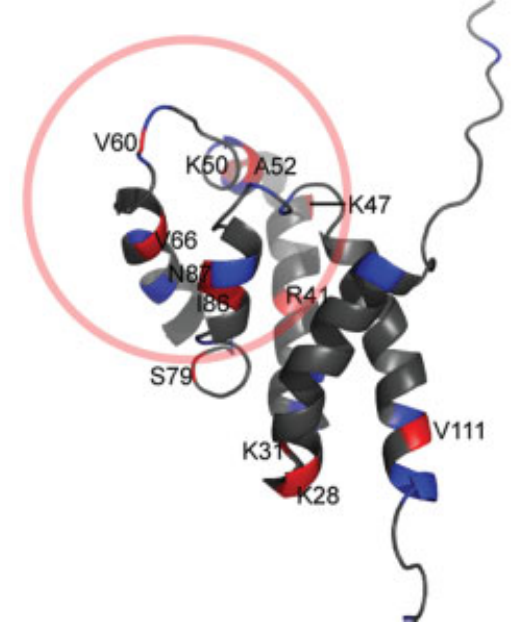

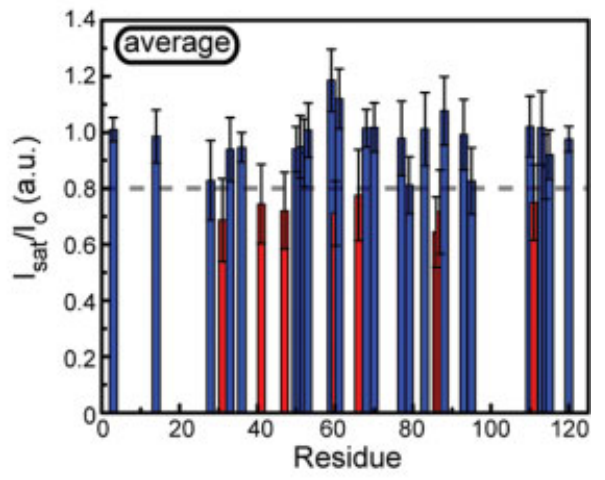

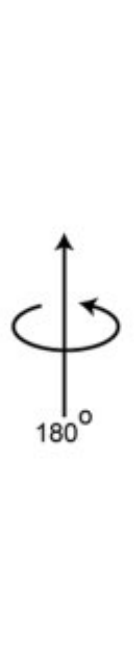

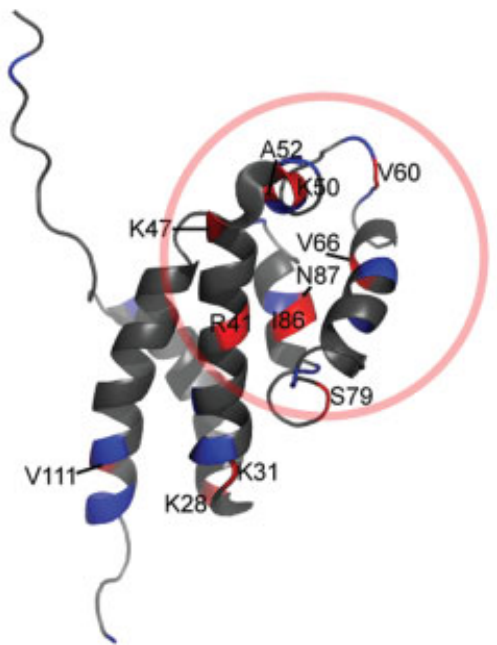

FIGURE 4 Intensity ratios $\left(I_{\text {sat }} / I_{\mathrm{o}}\right)$ from cross-relaxation experiments saturating protons at positions (7), (10), and (24) and their average is shown in (a). The 32 residues that were measured are color coded according to intensity reductions with those showing no significant reduction in intensity colored in blue and resonances showing intensity reductions greater than the threshold cutoff of 0.8 colored in red. The same color scheme is used in (b) with labeled residues being those that showed significant intensity reductions in any of the three sets of cross-relaxation data. Residues that could not be monitored are colored in gray and the shoulder region is circled in (b).

intensities in the on-resonance $\left(I_{\text {sat }}\right)$ versus off-resonance $\left(I_{\mathrm{o}}\right)$ experiment indicates cross-relaxation processes due to proximity of ligand protons to OSCP amide protons resulting from a specific binding interaction.

Although the cross-relaxation experiment is inherently insensitive since it recorded in $90 \% \mathrm{D}_{2} \mathrm{O}$ to avoid saturation of the water resonance, and despite the limited solubility of OSCP, which further limited sensitivity, 32 well-resolved resonances with sufficient signal:noise were observed in the OSCP120•1 complex. As shown in Figure 4, significant intensity reductions $(>20 \%)$ are observed for a number of residues. The majority of these residues (R41, K50, A52, V60, 


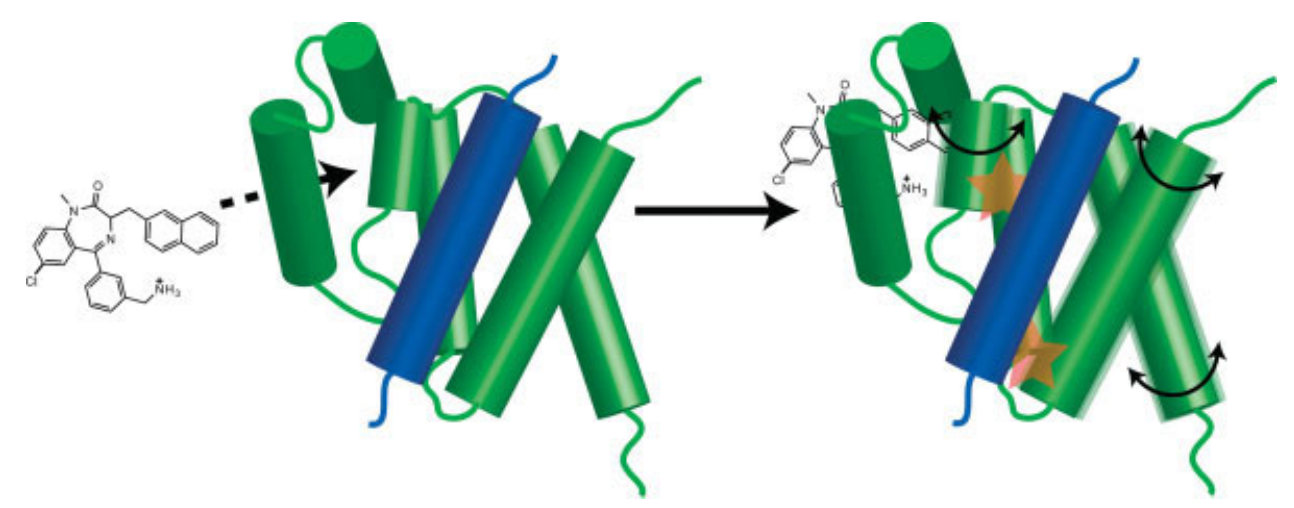

FIGURE 5 Proposed allosteric model where binding of 1 causes conformational rearrangements

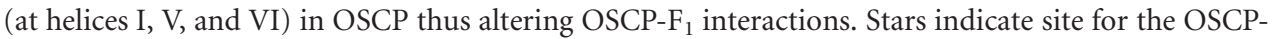
$\mathrm{F}_{1}$ peptide interaction that were perturbed during titration experiments with $\mathbf{1}$.

V66, S79, I86, and N87) fall in the "shoulder" region. Crossrelaxation to residues in the tail region (K28, K31, and V111) was also observed and V111 corresponds to a residue for which significant chemical shift perturbations upon titration of 1 were measured. However, this site is most likely cryptic and only present in the truncated OSCP constructs because the perturbations are diminished or otherwise significantly altered in OSCP145. Taken together, the chemical shift perturbation and cross relaxation studies are consistent with a unique binding site for 1 on the OSCP.

\section{DISCUSSION}

Inhibitors of the mitochondrial $\mathrm{F}_{1} \mathrm{~F}_{0}$-ATPase are powerful tools for probing the structure and function of the enzyme and like $\mathrm{Bz}-423$, some have therapeutic potential. ${ }^{25-27} \mathrm{~A}$ diverse group of molecules inhibit the enzyme by binding within the $F_{1}$ domain. Representative compounds here include the antibiotics aurovertin and efrapeptin, phytochemicals like resveratrol, and the naturally occurring peptide inhibitor, IF1. Aurovertin binds to $\beta_{\mathrm{TP}}$ and $\beta_{\mathrm{E}}$ states of the enzyme and is thought to function by preventing closure of the interfaces necessary for catalytic cycling between subunit conformational states. ${ }^{4,28-31}$ Efrapeptin interacts with the $\gamma$ and $\beta_{\mathrm{E}}$ subunits where it can block recharging after catalysis. ${ }^{28}$ Similarly, resveratrol inhibits the $\mathrm{F}_{1} \mathrm{~F}_{0}$-ATPase by binding between the $\gamma$ and $\beta_{\mathrm{TP}}$ subunits where it can block the rotation of the $\gamma$ subunit so that the catalytic cycle cannot progress. $^{32}$ IF1 inhibits ATP hydrolysis when the ability of the enzyme to synthesize ATP is compromised. IF1 is thought to function by hindering the closure of the $\alpha_{\mathrm{DP}}-\beta_{\mathrm{DP}}$ catalytic interface thereby blocking ATP hydrolysis. ${ }^{33}$ Several molecules such as the macrolide oligomycin inhibit the $\mathrm{F}_{1} \mathrm{~F}_{0^{-}}$ ATPase by binding to the embedded $\mathrm{F}_{0}$ domain. ${ }^{8}$ Biochemi- cal studies suggest that oligomycin binding blocks the flow of protons through the $\mathrm{c}$-subunit.

Here, we used NMR spectroscopy to investigate binding of a water-soluble $\mathrm{Bz}-423$ analog to OSCP constructs of varying length in an effort to define the binding site for these inhibitory benzodiazepines on the protein. The chemical shift perturbation data on both OSCP120 and OSCP145 together with crossrelaxation data for OSCP120 suggest that 1 most likely binds to the shoulder region in a pocket defined by residues M51, L56, K65, V66, K75, K77, and N92. The peak intensities of unbound OSCP120 suggest that both the shoulder and tail regions are more flexible relative to the rest of the protein, potentially allowing for local rearrangements to occur upon ligand binding (data not shown). While we cannot rule out a possible secondary binding site in OSCP120 involving the tail region, this interaction was diminished in OSCP145, indicating that interactions at this site are not likely relevant and probably result as a consequence of the truncation only present in the 120-amino acid long OSCP construct.

Several residues show chemical shift perturbations in the titration experiments but no significant cross-relaxation, which is most consistent with conformational changes on binding to $\mathbf{1}$. These residues include A25 and L88, which are thought to directly interact with the $\mathrm{F}_{1} \cdot{ }^{16,34} \mathrm{~L} 88$ along with other residues in its vicinity also shift when comparing free OSCP120 and OSCP145, even though the 25 amino acids are added to the C-terminal end of OSCP145. On the basis of the NMR structure of OSCP120, L88 is distant from the additional 25 amino acids. These data further support the hypothesis that this region of the OSCP is susceptible to conformational changes, which may be important for the function of the protein in the fully assembled enzyme and the activity of Bz-423 like inhibitors.

On the basis of our data, we propose an allosteric model for the inhibitory action of $\mathbf{1}$. Binding of $\mathbf{1}$ to the shoulder 
region of OSCP results in conformational rearrangements at a site that contacts the $\mathrm{F}_{1}$ domain of $\mathrm{F}_{1} \mathrm{~F}_{0}$-ATPase, which interferes with the rotary mechanism of catalysis (Figure 5). Allosteric communication between 1 and $F_{1}$ binding sites may be achieved by relative twisting motions of helices I and VI. Such a structural rearrangement could explain the perturbations observed in the middle region linking $\mathbf{1}$ and the $\mathrm{F}_{1}$ binding site.

Another group of benzodiazepines have been reported that are structurally similar to Bz-423. ${ }^{35}$ Unlike Bz-423, these compounds selectively inhibit ATP hydrolysis catalyzed by the mitochondrial $\mathrm{F}_{1} \mathrm{~F}_{0}$-ATPase and may have use as antiischemia drugs. Preliminary studies suggest that these benzodiazepines also function through binding to the OSCP. ${ }^{36}$ Together, these data highlight the important role the OSCP plays in regulating both the synthetic and hydrolytic the function of the mitochondrial $\mathrm{F}_{1} \mathrm{~F}_{0}$-ATPase. A better understanding of how these compounds interact with the OSCP and inhibit the enzyme should assist in further elucidating the function of the OSCP and may also provide additional opportunities for drug discovery.

We thank P. L. Toogood for the synthesis of 2. G.D.G. and A.W.O. acknowledge stock ownership and consulting compensation from a corporation that has licensed certain commercial rights to Bz-423.

\section{REFERENCES}

1. Bednarski, J. J.; Warner, R. E.; Rao, T.; Leonetti, F.; Yung, R.; Richardson, B. C.; Johnson, K. J.; Ellman, J. A.; Opipari, A. W.; Glick, G. D. Arthritis Rheum 2003, 48, 757-766.

2. Blatt, N. B.; Bednarski, J. J.; Warner, R. E.; Leonetti, F.; Johnson, K. M.; Boitano, A.; Yung, R.; Richardson, B. C.; Johnson, K. J.; Ellman, J. A.; Opipari, A. W.; Glick, G. D. J Clin Invest 2002, $110,1123-1132$.

3. Johnson, K. M.; Chen, X. N.; Boitano, A.; Swenson, L.; Opipari, A. W.; Glick, G. D. Chem Biol 2005, 12, 485-496.

4. Johnson, K. M.; Cleary, J.; Fierke, C. A.; Opipari, A. W.; Glick, G. D. ACS Chem Biol 2006, 1, 304-308.

5. Blatt, N. B.; Boitano, A. E.; Lyssiotis, C. A.; Opipari, A. W.; Glick, G. D. Free Radical Bio Med 2008, 45, 1232-1242.

6. Blatt, N. B.; Boitano, A. E.; Lyssiotis, C. A.; Opipari, A. W., Jr.; Glick, G. D. Biochem Pharmacol, doi: 10.1016/j.bcp.2009.05.025.

7. De Milito, A.; Iessi, E.; Logozzi, M.; Lozupone, F.; Spada, M.; Marino, M. L.; Federici, C.; Perdicchio, M.; Matarrese, P.; Lugini, L.; Nilsson, A.; Fais, S. Cancer Res 2007, 67, 5408-5417.

8. Devenish, R. J.; Prescott, M.; Boyle, G. M.; Nagley, P. J Bioenerg Biomembr 2000, 32, 507-515.

9. Pedersen, P. L.; Ko, Y. H.; Hong, S. J. J Bioenerg Biomem 2000, 32, 325-332.
10. Senior, A. E.; Nadanaciva, S.; Weber, J. BBA-Bioenergetics 2002, 1553, 188-211.

11. Walker, J. E.; Collinson, I. R. FEBS Lett 1994, 346, 39-43.

12. Rubinstein, J. L.; Walker, J. E. J Mol Biol 2002, 321, 613-619.

13. Walker, J. E.; Fearnley, I. M.; Gay, N. J.; Gibson, B. W.; Northrop, F. D.; Powell, S. J.; Runswick, M. J.; Saraste, M.; Tybulewicz, V. L. J. J Mol Biol 1985, 184, 677-701.

14. Wilkens, S.; Dunn, S. D.; Chandler, J.; Dahlquist, F. W.; Capaldi, R. A. Nat Struct Biol 1997, 4, 198-201.

15. Boitano, A.; Emal, C. D.; Leonetti, F.; Blatt, N. B.; Dineen, T. A.; Ellman, J. A.; Roush, W. R.; Opipari, A. W.; Glick, G. D. Bioorgan Med Chem Lett 2003, 13, 3327-3330.

16. Carbajo, R. J.; Kellas, F. A.; Yang, J. C.; Runswick, M. J.; Montgomery, M. G.; Walker, J. E.; Neuhaus, D. J Mol Biol 2007, 368, 310-318.

17. Walker, J. E.; Gay, N. J.; Powell, S. J.; Kostina, M.; Dyer, M. R. Biochemistry 1987, 26, 8613-8619.

18. Delaglio, F.; Grzesiek, S.; Vuister, G. W.; Zhu, G.; Pfeifer, J.; Bax, A. J Biomol NMR 1995, 6, 277-293.

19. Goddard, T. D.; Kneller, D. G.; Delano, W. L. SPARKY 3, University of California: San Francisco.

20. Carbajo, R. J.; Kellas, F. A.; Runswick, M. J.; Montgomery, M. G.; Walker, J. E.; Neuhaus, D. J Mol Biol 2005, 351, 824-838.

21. Piotto, M.; Saudek, V.; Sklenar, V. J Biomol NMR 1992, 2, 661-665.

22. Kupce, E.; Wagner, G. J Magn Reson Ser B 1995, 109, 329-333.

23. Delano, L. Pymol, Delano Scientific, San Carlos, California.

24. Takahashi, H.; Nakanishi, T.; Kami, K.; Arata, Y.; Shimada, I. Nat Struct Biol 2000, 7, 220-223.

25. Gledhill, J. R.; Walker, J. E. Biochem J 2005, 386, 591-598.

26. Hong, S.; Pedersen, P. L. Microbiol Mol Biol 2008, 72, 590-641.

27. Toogood, P. L. Curr Opin Chem Biol 2008, 12, 457-463.

28. Abrahams, J. P.; Buchanan, S. K.; Van Raaij, M. J.; Fearnley, I. M.; Leslie, A. G. W.; Walker, J. E. Proc Natl Acad Sci USA 1996, 93, 9420-9424.

29. Duser, M. G.; Zarrabi, N.; Cipriano, D. J.; Ernst, S.; Glick, G. D.; Dunn, S. D.; Borsch, M. EMBO J, doi: 10.1038/emboj.2009.213.

30. vanRaaij, M. J.; Abrahams, J. P.; Leslie, A. G. W.; Walker, J. E. Proc Natl Acad Sci Acad USA 1996, 93, 6913-6917.

31. Johnson, K. M.; Swenson, L.; Opipari, A. W., Jr.; Reuter, R.; Zarrabi, N.; Fierke, C. A.; Borsch, M.; Glick, G. D. Biopolymers 2009, 91, 830-840.

32. Gledhill, J. R.; Montgomery, M. G.; Leslie, A. G. W.; Walker, J. E. Proc Natl Acad Sci USA 2007, 104, 13632-13637.

33. Campanella, M.; Casswell, E.; Chong, S.; Farah, Z.; Wieckowski, M. R.; Abramov, A. Y.; Tinker, A.; Duchen, M. R. Cell Metab 2008, 8, 13-25.

34. Boyer, P. D. Annu Rev Biochem 1997, 66, 717-749.

35. Hamann, L. G.; Ding, C. Z.; Miller, A. V.; Madsen, C. S.; Wang, P.; Stein, P. D.; Pudzianowski, A. T.; Green, D. W.; Monshizadegan, H.; Atwal, K. S. Bioorg Med Chem Lett 2004, 14, 10311034.

36. Johnson, K. M. PhD Thesis, University of Michigan, 2005.

Reviewing Editor: Kenneth J. Breslauer 